\title{
Transportrecht in der Corona-Krise
}

\author{
Jun.-Prof. Dr. Andreas Maurer"
}

\section{Inhaltsübersicht}

A. Einleitung

B. Haftung bei pandemiebedingter Beschädigung oder verspäteter Ablieferung des

Transportguts

I. (Kurze) Einführung in das Transportrecht 208

1. Komplexität des Transportrechts 208

2. Grundzüge des deutschen Frachtrechts 210

II. Haftung wegen verspäteter Ablieferung durch den Frachtführer aufgrund der Corona-Krise

1. Lieferfristüberschreitung bei innerdeutschem Transport

2. Lieferfristüberschreitung bei Transport nach Polen

3. Lieferfristüberschreitung bei Transport nach Weißrussland (durch Polen als Transitland)

4. Zusammenfassung

1. Frachtrechtliche Regelung

2. Anwendbarkeit des allgemeinen Leistungsstörungsrechts 218

IV. Ersatz von Mehraufwendungen des Frachtführers

1. Standgeld/Vergütung von dem Risikobereich des Absenders zuzurechnenden Verzögerungen

2. Ersatz von Aufwendungen auf das Gut und die Ausführung von Weisungen durch den Auftraggeber

\section{A. Einleitung}

Als im Dezember 2019 erste Nachrichten vom vermehrten Auftreten einer Lungenkrankheit in China verbreitet wurden, schien kein Grund zu er-

* Der Autor ist Juniorprofessor für Bürgerliches Recht mit Schwerpunkt Transportrecht an der Universität Mannheim. 
höhter Vorsicht oder Sorge zu bestehen. Im Januar 2020 wurden die Nachrichten konkreter und ein neuartiger Erreger wurde als Ursache der Lungenerkrankung ausgemacht. Es handelte sich um einen Erreger, der ein schweres akutes respiratorisches Syndrom (SARS) hervorrief. Dieser Erreger wurde als Virus aus der Gruppe der Coronaviren identifiziert. Die Krankheit wurde daher mit dem Namen Corona-Virus Disease (CoViD) bezeichnet und weil sie im Jahr 2019 erstmals auftrat mit der Ordnungsnummer 19 versehen. Geboren war die Krankheitsbezeichnung CoViD-19, die die Welt in ein bis dahin nicht gekanntes Chaos stürzen sollte. Nachdem sich die Krankheit bis Mitte März des Jahres 2020 in aller Welt verbreitet hatte, sprach die WHO seit dem 11.03.2019 von einer Pandemie. $\mathrm{Zu}$ diesem Zeitpunkt waren in Europa bereits 20.000 bestätigte Fälle zu beklagen, von denen rund 1.000 tödlich ausgegangen waren. Um die Erkrankung einzudämmen, empfahlen Virologen und Epidemiologen strikte Kontaktsperren, die auf der ganzen Welt zu unterschiedlichen Zeiten umgesetzt worden waren. Einzelne Nationalstaaten riegelten ihre Grenzen ab, was teilweise so kurzfristig geschah, dass keine Reaktionszeit verblieb. Das führte nicht nur dazu, dass hunderttausende Urlauber und Geschäftsreisende im Ausland gestrandet waren, auch der Transport von Waren und Gütern war innerhalb kürzester Zeit massiv betroffen. Lieferketten brachen zusammen und transportierte Güter konnten wegen Grenzsperrungen oder Betriebsschließungen nicht ausgeliefert werden. Dies betraf oft auch hochwertige, verderbliche oder temperaturempfindliche Güter, so dass für die Ladung ein erheblich größeres Transportrisiko zum Beispiel durch Diebstahl hochwertiger Güter oder durch den Verlust verderblicher Waren zu verzeichnen war. ${ }^{1}$ Bereits abgeschlossene Transportverträge konnten nicht oder nur unter unverhältnismäßigem Aufwand durchgeführt werden, weil sich Spediteure und Frachtführer urplötzlich in erheblichen Pflichtenkollisionen sahen. Einerseits hatten sie Frachtaufträge zu erfüllen, andererseits bestanden Reisewarnungen des Auswärtigen Amtes. Arbeitnehmer, die einer Risikogruppe angehörten, konnten so vor dem Hintergrund von Fürsorgepflichten der Arbeitgeber kaum in Risikogebiete entsandt werden. Gleichzeitig ging die Nachfrage nach Transportleistungen in einigen Bereichen erheblich zurück. ${ }^{2}$

1 VerkehrsRundschau online, 22.04.2020, Frachtrisiken für Transportunternehmen und Versender nehmen erheblich zu, https:/www.verkehrsrundschau.de [Bezahlschranke] (zuletzt besucht am 27.04.2020).

2 VerkehrsRundschau online, 24.04.2020, Lkw-Verkehr in Italien um 37 Prozent rückläufig; VerkehrsRundschau online, 24.04.2020, Corona-Krise: Binnenschiffer spüren Flaute; VerkehrsRundschau online, 24.04.2020, Hamburger Spediteure kla- 
Die durch die Corona-Krise hervorgerufenen Verwerfungen in der Transportbranche sind teilweise wirtschaftlicher Natur, teilweise werfen sie aber auch konkrete rechtliche Fragen auf. Es geht zum Beispiel darum, wer dafür haften muss, wenn Waren wegen Grenz- oder Betriebsschließungen nicht rechtzeitig abgeliefert werden können. Aber auch viele Versender, die bereits in Vor-Corona-Zeiten Transportdienstleistungen eingekauft haben, werden sich fragen, ob und unter welchen Bedingungen sie vom Vertrag zurücktreten können. Schließlich ist auch für die Frachtführer interessant, ob sie eventuell entstandene Mehrkosten auf die Auftraggeber abwälzen können. Diese Probleme betreffen im Kern jeweils die Risikoverteilung zwischen den Vertragsparteien und die Frage, wie sich diese Risikoverteilung im Fall einer unvorhersehbaren Pandemie und deren Folgen verschieben könnte. Diese Problematik soll im Folgenden exemplarisch aufgeworfen und diskutiert werden. Die Liste der offenen Probleme ließe sich freilich fortsetzen. Im Fall einer Schiffscharter zum Beispiel stellt sich die Frage, wer für pandemiebedingte Liegezeiten aufkommt. Es stellt sich in einigen Fällen die zuvor bereits aufgeworfene Frage, ob bestimmte Mitarbeiterinnen und Mitarbeiter in der Transportbranche eingesetzt werden können, insbesondere, wenn sie einer Risikogruppe angehören. Viele Sachverhalte aus dem Bereich des Personentransports werden sich mit Problemen des Reiserechts überschneiden und werden an anderer Stelle in diesem Band besprochen.

\section{B. Haftung bei pandemiebedingter Beschädigung oder verspäteter Ablieferung des Transportguts}

Das Transportrecht gehört in Deutschland weder zum Prüfungsstoff in der ersten juristischen Prüfung noch zu demjenigen der zweiten juristischen Prüfung. Es wird an Universitäten zudem nur sehr vereinzelt gelehrt. Vor diesem Hintergrund und weil Kenntnisse des Transportrechts hier nicht vorausgesetzt werden sollen, ist es erforderlich, eine kurze Einführung in die zentralen Vorschriften und die zentrale Systematik des Transportrechts zu geben.

gen über hohen Rückgang im Export und Import, jeweils https://www.verkehrsru ndschau.de [Bezahlschranke] (zuletzt besucht am 27.04.2020). 


\section{I. (Kurze) Einfübrung in das Transportrecht}

\section{Komplexität des Transportrechts}

Das Transportrecht ist komplex in vielerlei Hinsicht. ${ }^{3}$ Anders als bei vielen anderen Vertragsbeziehungen, sind beim Transport regelmäßig bereits in der Standardfallgestaltung mindestens drei Parteien beteiligt: Der Absender, der Frachtführer und der Empfänger des Guts. Zwar ist der Empfänger nicht zwingend der Vertragspartner des Frachtführers, aber er kann regelmäßig im Rahmen der Transportabwicklung eigene Rechte gegen den Frachtführer geltend machen. So sieht $\$ 421$ Abs. 1 S. 2 HGB zum Beispiel als Fall der gesetzlich geregelten Drittschadensliquidation vor, dass der Empfänger in den Fällen des Verlusts, der Beschädigung oder der verspäteten Ablieferung des Guts eigene Ansprüche gegen den Frachtführer geltend machen kann. In den wenigsten Fällen wird es aber bei drei Beteiligten bleiben. Oft werden Frachtführer oder Spediteure weitere Unterfrachtführer einschalten, die ihrerseits wiederum Teil der umfangreichen Verflechtungen im Rahmen des Transportvertrags werden.

Das Transportrecht ist zudem international, und zwar in zweierlei Hinsicht. Einerseits ist der Transport selbst in den meisten Fällen grenzüberschreitend, andererseits ist das Recht des internationalen Warentransports geprägt von vielen internationalen Übereinkommen und europäischen Regeln, die in Regelungstiefe und Regelungsbreite sehr unterschiedlich sind. So wird der Schienentransport geregelt durch die Convention relative aux transports internationaux ferroviaires (COTIF) $)^{4}$, der Straßentransport durch die Convention relative au contrat de transport international de

3 Siehe hierzu zum Beispiel R. Freise, Das Transportrecht - Stand und Entwicklungslinien, RdTW 2013, S. 41 (41 ff.).

4 BGBl. 1985 II 130 ff. mit der Verordnung über die Inkraftsetzung des Übereinkommens vom 09.05.1980 über den internationalen Eisenbahnverkehr, BGBl. 1985 II $666 \mathrm{ff}$., in Kraft getreten am 01.05.1985, BGBl. 1985 II $1001 \mathrm{f}$. Aktuell gilt die COTIF in der Fassung des Änderungsprotokolls vom 03.06.1999 (BGBl. 2002 II 2140, 2142, 2149), zuletzt geändert durch die am 20. April 2016 im schriftlichen Verfahren des Revisionsausschusses der zwischenstaatlichen Organisation für den internationalen Eisenbahnverkehr (OTIF) beschlossenen Änderungen, die in Deutschland durch die dritte Verordnung zur Änderung des Übereinkommens über den internationalen Eisenbahnverkehr (COTIF) im Bundesgesetzblatt bekannt gemacht worden ist, BGBl. 2016 II $378 \mathrm{ff}$. 
marchandises par route $(\mathrm{CMR})^{5}$, die Binnenschifffahrt durch die Convention de Budapest relative au contrat de transport de marchandises en navigation intérieure $(\mathrm{CMNI})^{6}$, der Luftverkehr durch das Montrealer Übereinkommen $^{7}$ und der Seeverkehr durch gleich drei Übereinkommen, die jeweils in unterschiedlichen Ländern gelten und daher eher zu einer Fragmentierung des internationalen Seehandels führen als zu dessen Vereinheitlichung. ${ }^{8}$ Ein Übereinkommen zum Multimodalverkehr, zu Transporten also, die mit unterschiedlichen Verkehrsmitteln durchgeführt werden (Rotterdam Regeln), ist bis heute noch nicht in Kraft getreten, weil die hierfür erforderlichen 20 Ratifikationen noch nicht erfolgt sind, obwohl es bereits im Jahr 2008 verabschiedet worden war.

Wo einheitliche internationale Regeln fehlen, kommt wiederum nationales Recht zur Anwendung, wobei zum Beispiel Art. 6 ROM I VO eine eigene Kollisionsnorm für den Transportvertrag enthält. Schließlich verlässt sich die Transportbranche vielfach auf allgemeine Geschäftsbedingungen, die zumindest auch die Funktion haben, die Lücken des internationalen Einheitsrechts sowie nationaler Regelungen zu schließen.? Schließlich kommt hinzu, dass gerade das internationale Einheitsrecht des Warentransports eigene Regelungen für jeden einzelnen Verkehrsträger bereithält. ${ }^{10}$ All das macht das Transportrecht insgesamt ausgesprochen unübersichtlich. ${ }^{11}$

5 Ratifiziert in der Bundesrepublik Deutschland durch Gesetz vom 16.08.1961 (BGBl. 1961 II 1120 ff.) und in Kraft getreten am 05.02.1962 (Bekanntmachung vom 28.12.1961, BGBl. 1962 II S. 12).

6 In der Bundesrepublik Deutschland ratifiziert durch Gesetz vom 17.03.2007 (BGBl. 2007 II 298 ff.) und in Kraft getreten am 01.11.2007 (Bekanntmachung vom 3. August 2007, BGBl. 2007 II 1390).

7 Übereinkommen zur Vereinheitlichung bestimmter Vorschriften über die Beförderung im internationalen Luftverkehr vom 28. Mai 1999, (BGBl. 2004 II S. 458), in der Bundesrepublik Deutschland zuletzt geändert durch Art. 1 VO über die Inkraftsetzung der angepassten Haftungshöchstbeträge des Montrealer Übereinkommens vom 14.12.2009 (BGBl. 2009 II S. 1258).

8 Siehe dazu zum Beispiel J. Ramberg, Global Unification of Transport Law: A Hopeless Task?, Penn State International Law Review (2009) 851, 852 oder J. Basedow, Perspektiven des Seerechts, JZ 1999, S. 9 (13).

9 A. Maurer, Lex Maritima (2012), S. 42 ff.

10 Siehe dazu A. Maurer, Einheitsrecht im internationalen Warentransport, RabelsZ 81 (2017), S. 117 (127 ff.).

11 J. Basedow, Der Transportvertrag, Tübingen 1987, S. 3. 


\section{Grundzüge des deutschen Frachtrechts}

Neben der beschriebenen Komplexität des Transportrechts führt auch die eigentümliche Systematik seines Haftungsregimes dazu, dass es sich nicht ohne Weiteres auf Anhieb erschließt.

Die zentrale Haftungsnorm des deutschen Frachtrechts ist der $\$ 425$ HGB. Nach Absatz 1 dieser Vorschrift haftet der Frachtführer „für den Schaden, der durch Verlust oder Beschädigung des Gutes in der Zeit von der Übernahme zur Beförderung bis zur Ablieferung oder durch Überschreitung der Lieferfrist entsteht". Diese Vorschrift ist im Zusammenhang mit $\$ 426 \mathrm{HGB}$ zu sehen, nach dem der Frachtführer von der Haftung befreit ist, „soweit der Verlust, die Beschädigung oder die Überschreitung der Lieferfrist auf Umständen beruht, die der Frachtführer auch bei größter Sorgfalt nicht vermeiden und deren Folgen er nicht abwenden konnte“. Diese beiden Vorschriften bilden in ihrer Zusammenschau eine verschuldensunabhängige Haftung des Frachtführers, die sogenannte Obhutshaftung, die nur in denjenigen Fällen nicht eingreift, in denen der Frachtführer die eingetretene Pflichtverletzung auch bei größter Sorgfalt nicht vermeiden und die Folgen nicht abwenden konnte. ${ }^{12}$ Für den Zeitraum, in dem der Frachtführer das Gut in seiner Obhut hat, haftet er also zumindest grundsätzlich sehr umfassend und je nach seiner rechtlichen Organisationsform auch mit seinem gesamten Vermögen. Weil aber transportierte Güter oft sehr wertvoll sein können, wäre die umfassende Haftung des Frachtführers vor dem Hintergrund der verschuldensunabhängigen Haftung für Verlust, Beschädigung und Überschreitung der Lieferfrist ein enorm großes Risiko. ${ }^{13}$ Daher enthalten sowohl das deutsche Recht als auch internationale frachtrechtliche Übereinkommen eine Obergrenze für die Haftung des Frachtführers, die unabhängig vom Wert des zu transportierenden Guts eine Höchstgrenze für die Haftung des Frachtführers festlegen. Im deutschen Recht ist diese Haftungshöchstgrenze in $\$ 431$ HGB geregelt. Nach dieser Vorschrift ist die Haftung des Frachtführers in Fällen der Beschädigung oder des Verlusts des Guts begrenzt auf 8,33 Sonderziehungsrechte (SZR) pro Kilogramm des Rohgewichts des Guts. Ein Sonderziehungsrecht entspricht derzeit etwa $€ 1,26$. Für die Beschädigung oder den Verlust einer Sendung mit einem Rohgewicht von $10 \mathrm{~kg}$ würde der

$12 \mathrm{Zu}$ den Einzelheiten siehe zum Beispiel A. Maurer, in: H. Staub Großkommentar zum HGB, Berlin 2017, Band 12/1, $\$ 425$ HGB.

13 Zu Hintergründen siehe M. Paschke, in: Oetker, Handelsgesetzbuch, München 2019, \425, Rn. 1 f. 
Frachtführer also verschuldensunabhängig haften, jedoch nur bis zu einem Höchstbetrag von $€ 104,96$ (8,33 SZR x € 1,26 x 10 kg). Bei Verspätungsschäden beträgt die Haftungshöchstgrenze gemäß $₫ 431$ Abs. 3 HGB den dreifachen Betrag der Fracht, also der Vergütung des Frachtführers. Diese Haftungsgrenzen gelten gemäß $₫ 434$ HGB grundsätzlich auch für außervertragliche Ansprüche. In Internationalen Übereinkommen findet sich diese Haftungshöchstgrenze teilweise auch wieder (CMR), sie unterscheidet sich jedoch auch in einigen Übereinkommen. So gilt zum Beispiel im Luftverkehr eine Haftungsbegrenzung von 22 SZR $/ \mathrm{kg}$. Andere Übereinkommen stellen schließlich neben dem Gewicht zusätzlich auch auf einzelne Packstücke $a b$, für die dann zum Beispiel alternativ eine Haftungshöchstgrenze von 666,67 SZR gilt. Die Unterschiede können hierbei aber recht groß sein, was wiederum zur Komplexität des Transportrechts beiträgt. Von diesen Haftungsgrenzen kann in begrenztem Umfang abgewichen werden, $\mathbb{} 449$ HGB.

Schließlich kann sich der Frachtführer auf die Haftungsbegrenzung des $\$ 431$ HGB jedoch gemäß $\$ 435$ HGB nicht berufen, „wenn der Schaden auf eine Handlung oder Unterlassung zurückzuführen ist, die der Frachtführer $[\ldots]$ vorsätzlich oder leichtfertig und in dem Bewußtsein, daß ein Schaden mit Wahrscheinlichkeit eintreten werde, begangen hat“.

Vor diesem Hintergrund lässt sich nun das Haftungsregime des (deutschen) Transportrechts zumindest in sehr groben Zügen entfalten:

1.) Der Frachtführer haftet grundsätzlich verschuldensunabhängig für den Zeitraum zwischen Übernahme und Ablieferung des Guts (Obhutshaftung), $\$ 425$ HGB.

2.) Der Frachtführer haftet regelmäßig allerdings nur bis zu bestimmten Höchstbeträgen, $\$ 431$ HGB (abweichende Vereinbarungen unter den Voraussetzungen des $\$ 449$ HGB möglich).

3.) Die Haftungshöchstgrenzen entfallen zugunsten einer umfassenden unbegrenzten Haftung, wenn der Frachtführer (oder eine ihm zuzurechnende Person) vorsätzlich oder leichtfertig gehandelt hat, $\mathbb{} \$ 435$ HGB.

4.) Eine Haftung des Frachtführers ist ausgeschlossen, wenn er die Pflichtverletzung auch bei größter Sorgfalt nicht vermeiden und die Folgen nicht abwenden konnte, $\$ 426$ HGB. 


\section{Haftung wegen verspäteter Ablieferung durch den Frachtführer aufgrund der Corona-Krise}

Nach dieser holzschnittartigen Einführung in das Frachtrecht werden die grundlegenden Problemkonstellationen im Zusammenhang mit der Corona-Krise etwas klarer und die zu formulierenden Fragen deutlicher. Gleichzeitig bleiben die denkbaren Fallgestaltungen vielfältig. Schon allein der Umstand, dass ein LKW aufgrund einer Grenzschließung oder auch wegen umfangreicher Einreisekontrollen im Stau vor einem Grenzübergang steht, kann unterschiedliche Folgen haben. Führt die Verzögerung des Transports zu einer verspäteten Ablieferung des Guts, liegt eine Lieferverzögerung vor. Dies gilt selbst im Fall einer absoluten Fixschuld, denn aus transportrechtlicher Sicht kann das Gut auch nach Ablauf der Lieferfrist noch ausgeliefert werden. ${ }^{14}$ Ist das Gut aber verderblich und verdirbt während des Transports, weil die Auslieferung nicht rechtzeitig erfolgen konnte, liegt eine Beschädigung vor. ${ }^{15}$ Ist der Schaden eingetreten, weil der Betrieb des Empfängers geschlossen war oder weil sich die Ablieferung wegen der Einhaltung besonderer Hygienevorschriften im Betrieb des Empfängers verzögert hat, kann eine Schadensteilung gemäß $\$ 425$ Abs. 2 HGB infrage kommen. Da es hier oftmals entscheidend auf die Umstände des Einzelfalls ankommt, sollen im Folgenden einige Fallgestaltungen durchgespielt werden, die anhand eines immer wieder abgewandelten Falls erläutert werden sollen.

\section{Grundfall:}

Polen hatte am 14.03.2020, einem Samstag, angekündigt, ab dem darauffolgenden Sonntag seine Grenzen für alle Ausländer zu schließen. Später sollten dann umfangreiche Grenzkontrollen stattfinden. Menschen, die in Polen wohnen, sollten sich nach der Einreise aus Deutschland für $14 \mathrm{Ta}$ ge in Quarantäne begeben. Bereits kurz nach der Ankündigung bildeten sich an den Grenzübergängen nach Polen Staus, die teilweise eine Länge von teils mehr als 60 Kilometern erreicht und sich erst im Lauf der folgenden Woche wieder aufgelöst hatten. Obwohl der Güterverkehr weiter fließen durfte, standen einige LKW mehrere Tage in den entstandenen Staus;

14 Etwas anderes gilt möglicherweise dann, wenn die Verlustvermutung des $\$ 424$ HGB eintritt. Siehe zum Ganzen I. Koller, in: ders., Transportrecht Kommentar, München 2016, $\$ 425$ HGB, Rn. 9 a.

15 A. Maurer, in: Staub Großkommentar zum HGB, Band 12/1, Berlin 2017, $₫ 425$ HGB Rn. 21; Koller, Transportrecht, $\ 425$, Rn. 13. 
die Bundeswehr musste Reisende und Kraftfahrer mit Lebensmitteln versorgen. ${ }^{16}$

\section{Lieferfristüberschreitung bei innerdeutschem Transport}

Im Grundfall entstand der Stau am Grenzübergang nach Polen. Es ist aber gerade vor dem Hintergrund der Länge des Staus von mehr als $60 \mathrm{~km}$ durchaus denkbar, dass dieser Stau auch für Transporte zum Hindernis wurde, die innerdeutsch erfolgten und auf die daher deutsches Frachtrecht anwendbar wäre. ${ }^{17}$ Die erste Variante soll also einen Fall betrachten, in dem bei einem innerdeutschen Transport eine Lieferfristüberschreitung eingetreten ist, weil der LKW des Frachtführers im Stau vor der polnischen Grenze stand.

Nach den zuvor dargelegten Grundregeln der Haftung nach deutschem Frachtrecht würde der Frachtführer für einen durch die Überschreitung der Lieferfrist entstandenen Schaden haften. Es ist aber dann zu prüfen, ob der Haftungsausschluss gemäß $₫ 426$ HGB eingreift. Das wäre dann der Fall, wenn der Frachtführer die Lieferfristüberschreitung auch bei Anwendung größter Sorgfalt nicht hätte vermeiden können und ihre Folgen nicht hätte abwenden können.

Der hier anzulegende Sorgfaltsmaßstab ist sehr hoch und orientiert sich an der alten Fassung des $\$ 7$ Abs. 2 StVG, in dem die Ersatzpflicht des Halters eines KFZ ausgeschlossen war, wenn der Unfall durch ein „unabwendbares Ereignis“ (die aktuelle Fassung der Vorschrift verlangt höhere Gewalt) zurückzuführen war. ${ }^{18}$ In einer neueren Entscheidung formulierte das OLG Hamm:

„Der anzuwendende Sorgfaltsmaßstab orientiert sich, [...] in Anlehnung an $\$ 7$ Abs. 2 StVG a.F. an einem "idealen" Frachtführer, der eine über den gewöhnlichen Durchschnitt erheblich hinausgehende Aufmerksamkeit, Geschicklichkeit und Umsicht sowie ein geistesgegenwärtiges und sachgemäßes Handeln im Rahmen des Menschenmöglichen an den Tag legt. Der Fracht-

16 https:/www.mdr.de/sachsen/bautzen/goerlitz-weisswasser-zittau/stau-grenzepolen-a-vier-waechst-weiter-100.html (zuletzt besucht am 03.05.2020)

17 Zum Beispiel ein Transport von Dresden nach Görlitz.

18 BGH, VersR 1984, 551; BGH, VersR 1981, 1030. Auch wenn diese Entscheidungen zur CMR ergangen sind und fraglich ist, ob der Verschuldensmaßstab des deutschen Rechts auf das internationale Übereinkommen übertragbar ist, ist eine Auslegung der des deutschen Frachtrechts an diesem Maßstab durchaus legitim. Vgl. hierzu: Koller, Transportrecht, $₫ 426$ HGB, Rn. 4, Fn. 21. 
fübrer hat Anstrengungen zur Schadensverhütung bis zu dem Punkt zu erbringen, an dem diese bereits auf den ersten Blick als unzumutbar erscheinen. Das Vorliegen höherer Gewalt wird daher gerade nicht vorausgesetzt. "19

Rechtsprechung oder Literatur zu pandemiebedingten Lieferfristüberschreitungen liegen naturgemäß noch nicht vor. Allerdings hat sich die Kommentarliteratur mit der Frage auseinandergesetzt, wie Straßenblockaden zu behandeln seien, die der hier vorliegenden Grenzschließung durchaus ähnlich sind. Hierzu wird ausgeführt, dass ein sorgfältiger Frachtführer sich ständig über die Straßenlage informiere und Blockaden daher durch Umplanungen seiner Route umgehen könne. Eine Entlastung sei daher nur dann möglich, wenn das Ereignis, das zu einer Lieferfristüberschreitung führt, vollkommen überraschend aufgetreten ist und darauf durch Umplanungen nicht mehr reagiert werden kann. ${ }^{20}$

Es wird also für die Frage, ob sich der Frachtführer auf einen Ausschluss seiner Haftung gemäß $\$ 426$ HGB berufen kann darauf ankommen, ob er von dem Stau völlig überrascht wurde oder ob er noch die Möglichkeit einer Umplanung seiner Route gehabt hätte.

Kann er sich nicht auf einen Haftungsausschluss berufen, haftet der Frachtführer auf den Haftungshöchstbetrag des $\$ 431$ HGB. Die Haftungshöchstgrenzen würden gemäß $\$ 435$ HGB allerdings dann entfallen, wenn dem Frachtführer bei der Schadensverursachung Vorsatz oder Leichtfertigkeit in dem Bewusstsein, dass ein Schaden mit Wahrscheinlichkeit eintreten werde, zur Last fällt. Das wäre zum Beispiel dann denkbar, wenn der Frachtführer von dem Stau und seinem Ausmaß wusste und gleichwohl auf die Autobahn aufgefahren ist. In diesem Fall würde der Frachtführer dann ohne Haftungsbegrenzungen auf den vollen Schaden haften, der durch die Lieferfristüberschreitung entstanden ist.

\section{Lieferfristüberschreitung bei Transport nach Polen}

Auch bei der zweiten Fallvariante soll davon ausgegangen werden, dass der LKW eines Frachtführers an der Grenze zu Polen im Stau steht. Nun soll aber davon ausgegangen werden, dass der Transport für ein Ziel in Polen bestimmt war.

19 OLG Hamm, Urteil vom 21. April 2016 - I-18 U 17/14 -, Rn. 51, juris.

20 G. Kirchhof, in: BeckOK HGB, 27. Ed. 15.1.2020, HGB $₫ 426$ Rn. 4, 5; W. Schaffert, in: C. Ebenroth/K. Boujong/D. Joost/L. Strohn (Hrsg.), Handelsgesetzbuch, $\mathbb{\$} 426$ HGB, Rn. 3; Koller, Transportrecht, $₫ 426$ HGB, Rn. 6. 
Im Gegensatz zur ersten Variante handelt es sich nun also um einen grenzüberschreitenden Transport, was zur Folge hat, dass nicht deutsches Frachtrecht zur Anwendung kommt, sondern die CMR. Da das Haftungsregime des deutschen Frachtrechts der CMR in weiten Teilen angenähert ist, ${ }^{21}$ ist die grundsätzliche Haftung derjenigen des deutschen Frachtrechts sehr ähnlich. Die den $\$ \mathbb{S} 425$ und 426 HGB entsprechende Regelung findet sich in Artikel 17 CMR. Diese Vorschrift lautet:

\section{Art. 17}

(1) Der Frachtführer haftet für gänzlichen oder teilweisen Verlust und für Beschädigung des Gutes, sofern der Verlust oder die Beschädigung zwischen dem Zeitpunkt der Übernahme des Gutes und dem seiner Ablieferung eintritt, sowie für Überschreitung der Lieferfrist.

(2) Der Frachtführer ist von dieser Haftung befreit, wenn der Verlust, die Beschädigung oder die Überschreitung der Lieferfrist durch [...] Umstände verursacht worden ist, die der Frachtführer nicht vermeiden und deren Folgen er nicht abwenden konnte.

Ähnlich wie im deutschen Recht sind auch im Rahmen der CMR die Anforderungen an den Frachtführer sehr hoch. Der Haftungsmaßstab ist die äußerste zumutbare Sorgfalt eines besonders gewissenhaften Frachtführers. ${ }^{22}$ Auch hier wird es daher darauf ankommen, ob der Frachtführer eine Möglichkeit hatte, die Lieferfristüberschreitung zu verhindern. Gerade in diesem Fall dürfte das aber fraglich sein. Denn Polen hat nicht nur einen einzelnen Grenzübergang geschlossen, sondern alle Grenzübergänge. Es ist also ausgesprochen fraglich, ob überhaupt Maßnahmen denkbar gewesen wären, die eine Einhaltung der Lieferfrist ermöglicht hätten. Vor diesem Hintergrund kann es durchaus sein, dass Lieferfristüberschreitungen bei Transporten nach Polen, die aufgrund der Grenzschließungen eingetreten sind, nicht zu einer Haftung des Frachtführers führen, weil ein Haftungsausschluss im Sinne des Art. 17 Abs. 2 CMR vorgelegen hat. Das würde vor dem Hintergrund der Schließung aller Grenzen selbst dann gelten können, wenn der Frachtführer auf die dichte Autobahn aufgefahren ist, obwohl er durch Verfolgung des Verkehrsfunks oder durch andere Informationsquellen von dem Hindernis Kenntnis hätte haben können. Hierfür wird es aber entscheidend auf den Einzelfall ankommen.

21 BT-Drucks. 13/8445, S. 27.

22 F. Reuschle, in: Staub HGB, Berlin 2017, Band 14, Art. 17 CMR, Rn. 74. 


\section{Lieferfristüberschreitung bei Transport nach Weißrussland (durch Polen als Transitland)}

Die dritte Abwandlung soll nun einen Fall betreffen, in dem der LKW des Frachtführers in den Stau geraten ist, aber sein Ziel nicht in Polen liegt, sondern Polen nur als Transitland durchfahren werden sollte. Auch hier wäre die CMR und also Art. 17 CMR anwendbar. Der Unterschied zum Transport mit Ziel in Polen liegt darin, dass nun die Schließung der polnischen Grenzen nicht zwangsläufig dazu geführt hätte, dass ein Durchkommen zum Zielort nicht möglich gewesen wäre. Vielmehr wäre bei einem Transport, zum Beispiel nach Weißrussland, eine Umfahrung Polens, zum Beispiel durch Tschechien, die Slowakei und die Ukraine, möglich gewesen.

In diesem Fall wird es für die Frage, ob sich der Frachtführer auf einen Haftungsausschluss gemäß Art. 17 Abs. 2 CMR berufen kann, entscheidend darauf ankommen, ob er die Lieferverzögerung hätte vermeiden können. Das wäre gewiss dann nicht der Fall gewesen, wenn der LKW des Frachtführers die letzte Ausfahrt vor der Grenze bereits passiert hatte und sich der Stau erst dann gebildet hätte. Dann könnte er sich mit Erfolg auf einen Haftungsausschluss berufen. Wäre der LKW des Frachtführers aber in den bereits bestehenden Stau hineingefahren, obwohl er von dessen Existenz hätte wissen können und die Autobahn vorher hätte verlassen können, wird ihm ein Haftungsausschluss nicht zugutekommen können.

In letzterem Fall wäre der Frachtführer vielmehr verpflichtet gewesen, sich mit demjenigen in Verbindung zu setzen, der zur Verfügung über das Gut berechtigt ist und hätte von dieser Person Weisungen einholen müssen. Artikel 14 der CMR regelt hierzu:

„(1) Wenn aus irgendeinem Grunde vor Ankunft des Gutes an dem für die Ablieferung vorgesehenen Ort die Erfüllung des Vertrages zu den im Frachtbrief festgelegten Bedingungen unmöglich ist oder unmöglich wird, hat der Frachtführer Weisungen des nach Artikel 12 über das Gut Verfügungsberechtigten einzuholen. [...]“"

Würde der Frachtführer dann allerdings die Weisung erhalten, Polen zu umfahren, könnte er gemäß Art. 16 CMR die Erstattung zusätzlicher Kosten ersetzt verlangen. 


\section{Zusammenfassung}

Die Ausführungen zur Haftung bei Überschreitung der Lieferfrist haben gezeigt, dass sich eine pauschale Antwort auf die Frage der Frachtführerhaftung bei durch die Corona-Krise verursachten Lieferverzögerungen verbietet. Dabei kommt es nicht so sehr darauf an, ob deutsches Frachtrecht oder die CMR anwendbar ist, denn das HGB und die CMR halten sehr ähnliche Regelungen für die Haftung des Frachtführers bereit. Die zentrale Frage wird vielmehr sein, ob sich der Frachtführer auf einen Haftungsausschluss berufen kann, weil er die Lieferfristüberschreitung selbst bei Anwendung äußerster Sorgfalt nicht hätte vermeiden können. Ob das der Fall war, hängt von vielen unterschiedlichen Umständen $a b$ und wird daher nur im Einzelfall entschieden werden können. Schließlich wird auch entscheidend sein, welche vertraglichen Vereinbarungen die Vertragsparteien getroffen haben und ob sich daraus möglicherweise ein von den gesetzlichen Regeln abweichendes Ergebnis ableiten lässt.

\section{Kündigungsmöglichkeit für Auftraggeber/Absender}

Neben der Frage der Frachtführerhaftung hat sich für viele Absender und Auftraggeber aus ihrer Perspektive das Problem eröffnet, dass bereits gebuchte Transportdienstleistungen wegen der Corona-Krise nicht mehr benötigt werden.

\section{Frachtrechtliche Regelung}

Im deutschen Recht sieht $₫ 415$ HGB ein jederzeitiges Kündigungsrecht für den Absender vor. Kündigt der Absender nach dieser Vorschrift, sieht $\$ 415$ Abs. 2 HGB für den Frachtführer zwei Rechtsbehelfe vor: Er kann entweder die vereinbarte Fracht, etwaiges Standgeld und Aufwendungen ersetzt verlangen, wobei er sich ersparte oder böswillig nicht ersparte Aufwendungen anrechnen lassen muss, oder er kann ein Drittel der vereinbarten Fracht pauschal ohne konkreten Schadensnachweis als Kündigungsentschädigung verlangen. ${ }^{23}$ Die Rechtsbehelfe des Frachtführers stehen ihm jedoch dann nicht zu, wenn die Kündigung des Absenders auf Gründen

23 H. Merkt, in: A. Baumbach/K. Hopt/H. Merkt (Hrsg.), Handelsgesetzbuch, München 2020, \$415 Rn. 2. 
beruht, die dem Risikobereich des Frachtführers zuzurechnen sind, $\mathbb{} 415$ Abs. 2 S. 2 HGB.

\section{Anwendbarkeit des allgemeinen Leistungsstörungsrechts}

In der Literatur ist umstritten, ob neben der Kündigungsmöglichkeit des $\$ 415$ HGB auch das allgemeine Leistungsstörungsrecht anzuwenden ist, insbesondere die $\$ \$ 275 \mathrm{ff}$. und $323 \mathrm{ff}$. BGB. Diese Frage wird allerdings im Zusammenhang mit der Corona-Krise besonders wichtig. Denn Grenzschließungen, Betriebsschließungen, der Ausfall von Rohmateriallieferungen etc., die dazu führen könnten, dass der Absender einen bereits vereinbarten Transport nicht mehr durchführen kann oder möchte, fallen allesamt nicht in die Risikosphäre des Frachtführers. Für ihn würden also die Rechtsbehelfe des $₫ 415$ HGB bestehen bleiben und er könnte zumindest Teile der vereinbarten Vergütung geltend machen. Würde sich der Absender aber auf Unmöglichkeit berufen können, weil zum Beispiel der Ort, an dem das Gut aufgenommen oder abgeliefert werden soll, strengen Quarantänevorschriften unterliegt oder weil der Betrieb des Absenders oder des Empfängers behördlich untersagt wurde und daher eine Ablieferung des Guts nicht möglich wäre, könnte er sich gemäß $\$ 326$ Abs. 5, 323 BGB vom Vertrag lösen, ohne dass dem Frachtführer auch nur eine Teilvergütung zustehen würde. Zwar kämen Schadensersatzansprüche des Frachtführers gemäß $\$ 280$ BGB infrage, ${ }^{24}$ die aber ein Vertretenmüssen des Absenders voraussetzen, das in den hier interessierenden Fällen in der Regel nicht gegeben sein dürfte, so dass sich der Absender exkulpieren könnte mit der Folge, dass Schadensersatz nicht geschuldet wird. Der Absender würde also gerade bei Transporthindernissen, die nicht der Risikosphäre des Frachtführers zugerechnet werden können, im Frachtrecht schlechter gestellt als im allgemeinen Leistungsstörungsrecht. ${ }^{25}$

Die Debatte über die Anwendbarkeit des allgemeinen Leistungsstörungsrechts neben der Rücktrittsmöglichkeit des $\$ 415$ HGB geht auf den Hinweis in der Gesetzesbegründung zurück, wonach das allgemeine Leistungsstörungsrecht neben $\$ 415 \mathrm{HGB}$ anwendbar sein solle. ${ }^{26}$ In der Ursprungsfassung der Vorschrift im Zuge des Gesetzgebungsverfahrens ist $\$ 415$ HGB allerdings noch verändert worden, und es wurde die Risikover-

24 Koller, Transportrecht, $\mathbb{\$} 415$, Rn. 6.

25 Siehe dazu auch $P$. Schmidt, in: Staub HGB, Berlin 2014, $\$ 415$, Rn. 41.

26 BR-Drucks. 368/97, S. 45; BT-Drucks. 13/8445, S. 46. 
teilungsregel des $₫ 415$ Abs. 2 S. 2 HGB eingeführt, nach der ein Anspruch des Frachtführers nach Kündigung durch den Absender ausgeschlossen ist, wenn die Ursache der Kündigung im Risikobereich des Frachtführers liegt. Da mit $₫ 415$ Abs. 2 S. 2 HGB Elemente des allgemeinen Leistungsstörungsrechts in die Kündigungsregel aufgenommen worden seien, könne an der in der Regierungsbegründung geäußerten Auffassung zur parallelen Anwendbarkeit des allgemeinen Leistungsstörungsrechts nicht mehr festgehalten werden. ${ }^{27}$ Anders sieht dies zum Beispiel Reuschle, der mit Verweis auf die Regierungsbegründung von einer parallelen Anwendung des allgemeinen Leistungsstörungsrechts ausgeht. ${ }^{28}$ Dieser Streit hat sich mit der Einführung des $\$ 648$ BGB insbesondere für diejenigen Fälle entschärft, in denen der Werkvertrag und damit also auch der Frachtvertrag aus wichtigem Grund gekündigt werden kann, wenn die Voraussetzungen der Norm vorliegen. ${ }^{29}$ Voraussetzung dieser Norm ist allerdings, dass der Werkvertrag bereits in Vollzug gesetzt ist. Es verbleiben als problematische Fälle also nur solche, bei denen das Leistungshindernis und damit ein potentieller Kündigungsgrund des Absenders zu einem Zeitpunkt eintreten, in dem der Frachtvertrag noch nicht in Vollzug gesetzt war. Für solche Fälle wirft die Corona-Krise ein neues Licht auf die Frage der Risikoverteilung. Denn vor dem Hintergrund, dass Leistungshindernisse aufgrund der Corona-Krise regelmäßig keiner der Risikosphären der Vertragsparteien des Frachtvertrags zugeordnet werden können, wird deutlich, dass die Risikoverteilung des $₫ 415$ HGB allein zu unbilligen Ergebnissen führt und ein Rückgriff auf das allgemeine Leistungsstörungsrecht erforderlich ist. So muss davon ausgegangen werden, dass in Fällen, in denen vor Übernahme des Guts durch den Frachtführer bereits ein Leistungshindernis vorgelegen hat, eine Berufung des Absenders auf Unmöglichkeit möglich ist. ${ }^{30}$

27 K.-H.Thume, in: Münchener Kommentar zum HGB, München 2020, $\$ 415$ Rn. 35. Von einer "grundsätzlichen Subsidiarität“ des allgemeinen Leistungsstörungsrechts geht auch $P$. Schmidt, in: Staub HGB, $\mathbb{S} 415$, Rn. 42 aus.

28 F. Reuschle, in: in: Ebenroth/Boujong/Joost/Strohn (Hrsg.), Handelsgesetzbuch, $\$ 415$ Rn. 23 ff.; etwas differenzierter, aber grundsätzlich in ähnlicher Richtung Koller, Transportrecht, 9. Aufl. $\$ 415$ Rn. 3 ff., der vorliegende „Überschneidungen“ mit dem allgemeinen Leistungsstörungsrecht differenziert aufzulösen versucht.

29 K.-H. Thume, in: MüKoHGB, $\$ 415$ Rn. 37.

30 In diese Richtung auch Koller, Transportrecht, $\mathbb{\$} 415$, Rn. 6. Ebenso trotz grundsätzlicher Bedenken in Bezug auf die Anwendbarkeit des allgemeinen Leistungsstörungsrechts K.-H. Thume, in: MüKoHGB, $\mathbb{} 415$ Rn. 35. 


\section{Ersatz von Mehraufwendungen des Frachtführers}

Für viele Frachtführer stellt sich im Rahmen der Corona-Krise die Frage, ob sie zusätzliche Aufwendungen, die zum Beispiel durch längere Wartezeiten, die Umsetzung von Hygieneplänen etc. entstehen, ersetzt verlangen können.

Grundsätzlich ist die Risikoverteilung im Frachtrecht klar geregelt. Der Frachtführer ist verpflichtet, das Gut an den Bestimmungsort zu liefern und an den Empfänger abzuliefern, während der Absender verpflichtet ist, die vereinbarte Fracht (Vergütung) zu zahlen, $\$ 407$ HGB. Der Frachtführer ist also gehalten, bis zur Grenze des $\$ 275$ BGB alles zu tun, um seine Pflicht zu erfüllen. Allerdings kann der Frachtführer in einigen eng umrissenen Fällen Ersatz seiner zusätzlichen Aufwendungen oder eine zusätzliche Vergütung verlangen.

\section{Standgeld/Vergütung von dem Risikobereich des Absenders zuzurechnenden Verzögerungen}

Gemäß $₫ 412$ Abs. 1 HGB ist das Beladen und Entladen grundsätzlich Aufgabe des Frachtführers. Gemäß $₫ 412$ Abs. 2 HGB kann für die Lade- und Entladezeit keine gesonderte Vergütung verlangt werden. Etwas anderes gilt gemäß $\ 412$ Abs. 3 HGB allerdings dann, wenn der Frachtführer auf Grund vertraglicher Vereinbarung oder aus Gründen, die nicht seinem Risikobereich zuzurechnen sind, über die Lade- oder Entladezeit hinaus wartet. In diesem Fall hat er einen Anspruch auf eine angemessene Vergütung, die als Standgeld bezeichnet wird.

Gemäß $₫ 420$ Abs. 4 HGB kann der Frachtführer zusätzlich zur vereinbarten Fracht eine angemessene Vergütung verlangen, wenn nach Beginn der Beförderung und vor Ankunft an der Ablieferungsstelle eine Verzögerung eintritt, die dem Risikobereich des Absenders zuzuordnen ist.

Der Gesetzgeber geht in diesen und in weiteren Vorschriften davon aus, dass Störungen der Vertragsdurchführung stets dem Risiko- und Zurechnungsbereich zugeordnet werden könnten. Im Störungsfall sei dann jeweils nur das Schutzbedürfnis desjenigen Vertragspartners anerkennenswert, dessen Gefahrenkreis die Störungsursache nicht zuzuordnen ist. ${ }^{31}$ Während diese Zuordnung auf den ersten Blick einleuchtend erscheint, hat sich um die Frage, welche Risiken der Sphäre des Frachtführers oder 
des Absenders zuzuordnen seien, eine umfangreiche Debatte entwickelt. ${ }^{32}$ Dabei wird insbesondere kritisiert, dass der Gesetzgeber die sogenannte „neutrale Sphäre“ nicht hinreichend berücksichtigt habe, Ereignisse also, die keiner der beiden Parteien sinnvollerweise zugerechnet werden können. ${ }^{33}$

Bei Störungen aufgrund der Corona-Krise dürfte es sich in der Tat um solche handeln, die regelmäßig keiner der Parteien zugerechnet werden können. So bleibt es für den Frachtführer dabei, dass ein Warten aufgrund einer verzögerten Abwicklung im Betrieb des Empfängers auch dann nicht seiner Risikosphäre zuzuordnen ist, wenn sie ebenfalls nicht der Risikosphäre des Empfängers zuzuordnen ist. Vergleichbare Fälle wurden auch zuvor bereits so beurteilt, dass der Frachtführer Standgeld verlangen konnte. ${ }^{34}$ Eine verzögerte Abwicklung wegen fehlenden Personals auf Seiten des Empfängers dürfte jedenfalls nicht zur Risikosphäre des Frachtführers zuzurechnen sein. Eine Vergütung gemäß $\$ 420$ Abs. 4 HGB wird der Frachtführer wegen Störungen, die durch die Corona-Krise verursacht wurden, aber regelmäßig nicht verlangen können, denn solche Störungen werden typischerweise nicht dem Risikobereich des Absenders zuzuordnen sein.

\section{Ersatz von Aufwendungen auf das Gut und die Ausführung von Weisungen durch den Auftraggeber}

Gemäß $₫ 420$ Abs. 1 S. 2 HGB kann der Frachtführer Ersatz von Aufwendungen auf das Gut verlangen, die er für erforderlich halten durfte. Ein solcher Ersatzanspruch dürfte jedenfalls in Fällen entstanden sein, die in den zuvor als Beispiel gewählten Staus an der polnischen Grenze vorgekommen sind. Fahrer von Viehtransportern mussten teilweise Futter für die Tiere beschaffen, um deren Verhungern zu vermeiden. Eine solche

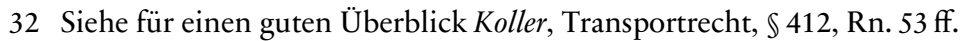

33 So zum Beispiel auch Reuschle, in: Ebenroth/Boujong/Joost/Strohn (Hrsg.), Handelsgesetzbuch, $\mathbb{} 412$ Rn. 37 f. mit Verweis auf C.-W. Canaris, Handelsrecht, $\mathbb{} 31$ Rn. 52.; siehe auch P. Schmidt, in: Staub HGB, $\$ 412$, Rn. 55. Einschränkend K.-H. Thume, in: Münchener Kommentar zum HGB, $\$ 412$ Rn. 43, der eine neutrale Sphäre nur dort erkennen will, wo der störende Einfluss gänzlich ohne Bezug zum Verkehrsgeschehen ist. Eine neutrale Sphäre ablehnend hingegen T. Braun, Das frachtrechtliche Leistungsstörungsrecht nach dem Transportrechtsreformgesetz, Münster 2002, S. 117.

34 Siehe zum Beispiel Koller, Transportrecht, $\mathbb{S} 412$, Rn. 57 mit verschiedenen Beispielen. 
Aufwendung wird im Rahmen des $₫ 420$ Abs. 1 S. 2 HGB jedenfalls ersatzfähig sein.

Auch kann der Frachtführer gemäß $\ 418$ Abs. 1 S. 4 HGB eine zusätzliche Fracht verlangen, wenn er Weisungen des Auftraggebers ausgeführt hat und ihm dadurch Mehrkosten entstanden sind. Hier ist insbesondere an Fälle zu denken, in denen der Auftraggeber die Weisung erteilt hat, erhebliche Umwege zu fahren, um die Güter zum Ziel zu bringen. Als Beispiel kann ebenfalls das zuvor bereits eingebrachte Beispiel genannt werden, in dem Polen durch Tschechien, die Slowakei und die Ukraine umfahren wurde, um Güter in Weißrussland abzuliefern.

\section{Zusammenfassung und Ausblick}

Die Transportbranche ist durch die Corona-Krise in vielerlei Hinsicht betroffen. Hier ging es darum, einige Fragen anhand eines Beispiels zu erörtern, wobei freilich weder die aufgeworfenen Fragen noch die Antworten abschließend sein können.

Auch wenn eine ganze Reihe von neuen Fragen durch die Corona-Krise aufgeworfen worden sind, ist das Transportrecht an sich recht gut ausgestattet, adäquate Lösungen zur Verfügung zu stellen. Sicherlich wird es darüber hinaus in einigen Fällen klare Antworten nicht geben können. Das liegt jedoch nicht etwa an den Eigenheiten der Corona-Krise, sondern vielmehr an der Struktur des Transportrechts, das sich bei der Zuweisung diverser Risiken nicht immer klar und eindeutig verhält, sondern im Einzelfall angemessene Lösungen erlaubt. So wird es auch bei der juristischen Aufarbeitung von Problemen, die auf der Corona-Krise beruhen, in den allermeisten Fällen um Lösungsansätze gehen müssen, die den konkreten Gegebenheiten des Einzelfalls Rechnung tragen. 